\title{
La investigación científica en la UNAH-VS: pasado, presente y futuro
}

Mirta Macías Ruano ${ }^{1}$

En la presente edición del Portal de la Ciencia, se abordará el tema de la investigación científica, tema de vital importancia en la vida académica, puesto que es el medio que permite a los estudiantes entender por qué y cómo suceden las cosas que los rodean con un propósito definido: mejorar la calidad de vida de todos los seres humanos. En tal sentido, es preciso señalar que la investigación científica va más allá de satisfacer la curiosidad, ya que contribuye a desarrollar el conocimiento sobre el funcionamiento de muchos temas vinculados a la salud y el trabajo, entre otros, tales como determinar la relación entre la cultura organizacional y el desempeño laboral, el autocuidado en el manejo de pleurovac y ejercicio respiratorio en pacientes de cirugía y el uso de nuevas tecnologías en el proceso electoral hondureño.

Pero, ¿qué se entiende por investigación científica? La respuesta indica que es un proceso complejo de aplicar el método científico en la investigación, cuyo resultado es el avance científico para resolver problemas o explicar determinados fenómenos observados o analizados. En nuestro caso, la investigación científica se respalda en el modelo teórico educativo de la UNAH-VS (2008:13).

Aunque la investigación científica plantea grandes retos dentro del contexto hondureño, en la UNAH-VS se han obtenido logros significativos en esta materia; por ejemplo, la organización del primer congreso de investigación científica, con una producción propia de 61 (85.9\%) trabajos de un total de 71 , de las distintas áreas del conocimiento, enmarcados en las prioridades de investigación de nuestra Alma Máter.

También se ha trabajó en la creación de dos unidades de investigación, odontología y enfermería; se han sistematizado y publicado aproximadamente 11 trabajos de investigación, producto de las asignaturas relacionadas con la investigación y cuyos estudiantes han sido asesorados por docentes. De igual forma, se asistió al 6 Congreso de Investigación Científica con una ponencia y en el 7 Congreso con un póster elaborado por los alumnos de la carrera de Economía.

En la parte de formación del recurso humano en investigación, se capacitó en la metodología de la investigación a estudiantes de la carrera de Derecho, en el marco

\footnotetext{
${ }^{1}$ Coordinación Regional de Investigación UNAH-VS. Dirección electrónica: mirtamaru@hotmail.com
} 
del Proyecto Semilleros de Investigación; igualmente se impartieron cinco cursos sobre investigación y un curso sobre propiedad intelectual y derechos de autor.

Pero, a pesar de los logros, se han presentado problemas en esta materia. Uno de estos fue la falta de reconocimiento de la Coordinación Regional de Investigación (CRI) en respuesta, para lograr visibilizar esta estructura educativa, la CRI realizó un proceso de socialización con los docentes y autoridades universitarias; posteriormente, se comenzaron a difundir las actividades que realiza esta Coordinación.

Con respecto a los docentes, estos tienen un papel protagónico en tarea universitaria, porque ellos son los que forman el recurso humano que estudia en UNAH-VS y en materia de investigación, son los encargados de crear las competencias en la actividad científica. Es decir, son los responsables de formar universitarios con capacidades analíticas, con criterio crítico y propositivos con respecto a la problemática que enfrenta la sociedad de la UNAH-VS, con la finalidad de aportar a la toma de decisiones para la solución de los problemas planteados.

Los docentes, a través de la asignatura de investigación, establecen el vínculo entre docencia e investigación, actividad que tiene incidencia en dos vías. Por un lado, contribuyen a la función social de la Universidad, o sea, formar recurso que tomará decisiones, contribuirá a la solución de los problemas del Valle de Sula o del entorno donde se encuentren; y por otro lado, permiten que la actividad científica se consolide y haya retroalimentación entre docencia e investigación.

Al respecto, los estudiantes se han mostrado muy dispuestos a participar en la actividad científica, pues aunque son voluntarios, han iniciado el Proyecto Semilleros de Investigación, con la participación de las carrera de Derecho y Economía y se espera ampliarlo a otras áreas del conocimiento. En este aspecto las experiencias han sido muy buenas, con resultados satisfactorios, tal como lo demuestran las publicaciones realizadas a la fecha, que su mayoría han sido realizadas como producto de las investigaciones de los alumnos.

Actualmente, la UNAH-VS sigue teniendo dentro de sus objetivos el avance de la investigación científica, para cuyo fin está realizando las siguientes gestiones: se continua con el proceso de socialización de la oferta que brinda la CRI, se están consolidando las unidades de gestión y creando otras nuevas, se motiva continuamente a los alumnos para que apliquen a becas de investigación como un incentivo para realizar la actividad científica, se hace la promoción de la CRI con jefes de departamento, coordinadores y claustro de profesores, a fin de lograr el empoderamiento de la CRI y su quehacer. 
En lo concerniente al futuro, la UNAH-VS se plantea los siguientes retos:

1. Establecer la estructura de investigación en UNAH-VS, para lo cual se precisa crear unidades en aquellas carreras donde aún no existen y consolidar las ya existentes.

2. Crear los grupos de investigación, pero para lograrlo es necesario organizar a los investigadores en grupos entre las distintas áreas del conocimiento, para sistematizar el portafolio de investigadores y precisar la oferta de investigadores con que cuentala UNAH-VS.

3. Capacitar el recurso humano en investigación científica, a fin de generar las competencias investigativas.

4. Divulgación de los resultados de investigación en versión digital y física (revista).

5. Consolidación del Congreso de Investigación como una tradición y como un medio de difusión de los resultados de la actividad científica.

6. Posicionar a la UNAH-VS como referente en investigación en zona del Valle de Sula. 